\title{
Laboratory experiments of particle size effect in X-ray fluorescence and implications to remote $\mathrm{X}$-ray spectrometry of lunar regolith surface
}

\author{
Yoko Maruyama $^{1,2}$, Kazunori Ogawa ${ }^{1,3}$, Tatsuaki Okada ${ }^{1,4}$, and Manabu Kato ${ }^{1,2,3}$ \\ ${ }^{1}$ Institute of Space and Astronautical Science, Japan Aerospace Exploration Agency, \\ 3-1-1 Yoshinodai, Sagamihara, Kanagawa 229-8510, Japan \\ ${ }^{2}$ Department of Earth and Planetary Science, University of Tokyo, 7-3-1 Hongo, Tokyo 113-0033, Japan \\ ${ }^{3}$ Department of Earth and Planetary Sciences, Tokyo Institute of Technology, Ookayama, Tokyo 152-8550, Japan \\ ${ }^{4}$ Department of Space and Astronautical Science, Sokendai, Sagamihara, Kanagawa 229-8510, Japan
}

(Received March 30, 2007; Revised October 29, 2007; Accepted October 29, 2007; Online published April 9, 2008)

\begin{abstract}
We performed laboratory experiments to investigate the effect of particle size in X-ray fluorescence (XRF) with respect to interpreting remote X-ray spectrometry. To simulate microscopic roughness of the uppermost surface of lunar regolith, we used powdery specimens of crashed rocks ranging 25 to $500 \mu \mathrm{m}$ in size and flat rock plates for comparison. Our results show that XRF intensities from powdery specimens decrease relative to those from flat plates by up to $50 \%$, especially for larger particle size and at increasing source-to-surface-to-detector angle (phase angle). Corrections should be required for elemental analysis by XRF spectrometry in the SELENE "Kaguya" and other planetary orbiter missions.
\end{abstract}

Key words: X-ray fluorescence, particle size effect, regolith, elemental composition, phase angle.

\section{Introduction}

Detailed studies of X-ray fluorescence (XRF) experiments on rough surfaces are required in order to be able to interpret the results of remote planetary X-ray spectrometry and determine the surface elemental composition with improved accuracy.

$\mathrm{XRF}$ is a well-established technique for major elemental analysis in the laboratory. Irradiation by primary X-rays excites atoms in the target material via photo-absorption. This is accompanied by the escape of inner-shell electrons, followed by the movement of an outer-shell electron to the vacancy. The result is the emission of X-rays characteristic of the element. In the same manner, the irradiation of atmosphere-free planetary surfaces by solar X-rays produces XRF excitation characteristic of elements. Major elemental mapping in planets can be carried out with concurrent observation of X-rays from the Sun and the planet, as was proven in the Apollo 15 and 16 missions (e.g., Adler and Trombka, 1977). This method has also been used in planetary missions such as the Near-Earth Asteroid Rendezvous-Shoemaker (Trombka et al., 2000; Nittler et al., 2001), Hayabusa (Okada et al., 2006, 2007), and SMART-1 (Kellett et al., 2006; Grande et al., 2007).

In the laboratory, flat samples or compacted powders are usually used as specimens. The surface roughness of these specimens is much less than $10 \mu \mathrm{m}$. However, planetary surfaces are covered with soils and breccias called regolith whose average diameter ranges from ten to hundreds of micrometers, far from the ideal surface for detailed anal-

Copyright (c) The Society of Geomagnetism and Earth, Planetary and Space Sciences (SGEPSS); The Seismological Society of Japan; The Volcanological Society of Japan; The Geodetic Society of Japan; The Japanese Society for Planetary Sciences; TERRAPUB ysis. Although particle size effect in XRF has been studied in a number of practical cases (Claisse and Samson, 1962; Suortti, 1972; Nielson and Rogers, 1986; Abdunnabi and Wasilewska-Radwanska, 1994), few studies have been carried out for planetary XRF spectrometry. Kuwada et al. (1997) performed laboratory experiments using a sandy specimen of having a simple composition, such as $\mathrm{SiC}$ and $\mathrm{Al}_{2} \mathrm{O}_{3}$ powders, and showed a remarkable decrease in XRF intensities when observed at rougher particle size and at larger phase angles. These researchers also carried out numerical calculations in which they assumed the rough surface was rectangular in shape. Their results were in good agreement with the experimental results, suggesting that the effects are mainly explained by the shadowing of incident primary X-rays and the absorption of emission fluorescent X-rays. They also pointed out that this decrease could cause a serious error in terms of rock-type classification by remote planetary missions, particularly when observed at large solar phase angles. Okada (2004) performed laboratory experiments under atmosphere (normal temperature and pressure) using a basaltic powder specimen and showed a clear decrease in $\mathrm{Ca} / \mathrm{Fe}$ and $\mathrm{Ti} / \mathrm{Fe}$ at larger phase angles, as expected based on the results of these earlier studies (Kuwada et al., 1997; Kuwada, 1998). XRF numerical modeling for regolith surface has been recently studied using a fractionalBrownian-motion model (Näränen et al., 2007). The results suggest that further detailed studies are required to investigate this effect.

In the SELENE (Selenological and Engineering Explorer, nicknamed "Kaguya", which means the lunar princess in a Japanese fairy tale) lunar polar orbiter mission and other future planetary missions, remote XRF spectrometry is planned at various solar phase angles (e.g., Okada et 

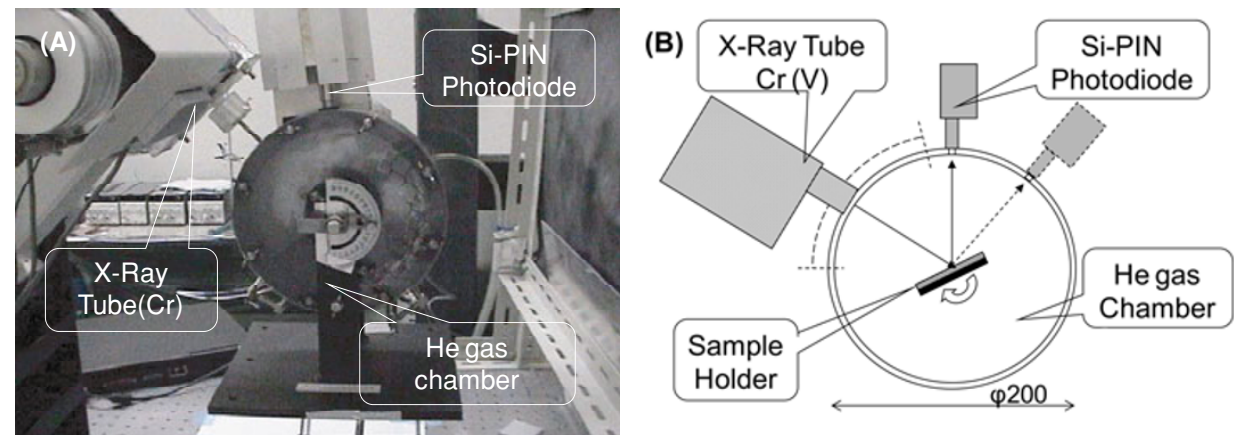

Fig. 1. The experimental setup (A) and its configuration (B) are shown. The specimens are placed on the sample holder inside the helium gas-filled chamber. The holder can be declined up to $30^{\circ}$. X-ray incident and emission angles are selectable by changing the attached position of $\mathrm{X}$-ray tube and detector.

al., 2002). It is therefore essential to investigate the particle size effect in XRF in more detail.

The aim of this study was to thoroughly investigate the angular dependency of XRF intensity from a rough surface and addresses the importance of this effect, especially for the X-ray Spectrometer (XRS) experiments onboard the SELENE orbiter (Okada et al., 2002; Shirai et al., 2008).

\section{Laboratory Experiments on the Particle Size Effect in XRF}

We performed laboratory experiments to investigate the particle size effect in XRF (Maruyama et al., 2006, 2007; Maruyama, 2007). Although the experimental setup should preferably be constructed under vacuum in order to avoid any absorption of low-energy XRF photons of all the major elements, we adopted a helium-filled condition because of its convenience in sample exchange, high X-ray transparency, and ease of source-to-sample-to-detector angle (phase angle) variation.

We developed an apparatus consisting of a helium chamber with incident and emission X-ray windows, an X-ray tube, and an X-ray detector (Fig. 1). X-ray incident and emission angles are changeable from $0^{\circ}$ (normal to the specimen's surface) to $\pm 90^{\circ}$, but there is a geometrical limitation of the minimum phase angle to $25^{\circ}$. Powdery and flat plate specimens are mounted on the sample holder inside the helium-filled chamber. The holder can be inclined up to $30^{\circ}$. The primary X-rays, generated using a chromiumtarget X-ray tube with a vanadium thin filter, consist of an intense line spectrum of $\mathrm{Cr}-\mathrm{K} \alpha(5.41 \mathrm{KeV})$ and the continuum spectra by bremsstrahlung emission. The beam diameter of the primary X-rays is collimated to $5 \mathrm{~mm}$ at the surface of specimen. XRF from the specimen is excited by irradiation of primary X-rays, detected with a Si-PIN photodiode (AMPTEK XR-100CR), and conducted by pulse height analysis from a multi-channel analyzer (NAIG E-551 and E-562A)-based spectrometer. The energy resolution of the detector $(200 \mathrm{eV}$ at $5.9 \mathrm{KeV})$ is sufficient to discriminate the XRF of each major element and observe these simultaneously. In this setup, line spectra of $\mathrm{Al}, \mathrm{Si}, \mathrm{Ca}, \mathrm{Ti}$, and $\mathrm{Fe}$ are detected with sufficiently high sensitivity, but the $\mathrm{Mg}$ line spectrum is too faint to analyze in the integration period of $600 \mathrm{~s}$. The intensities of the $\mathrm{K}-\alpha$ and $\mathrm{K}-\beta$ XRF lines are derived by Gaussian fitting of X-ray energy spectra.

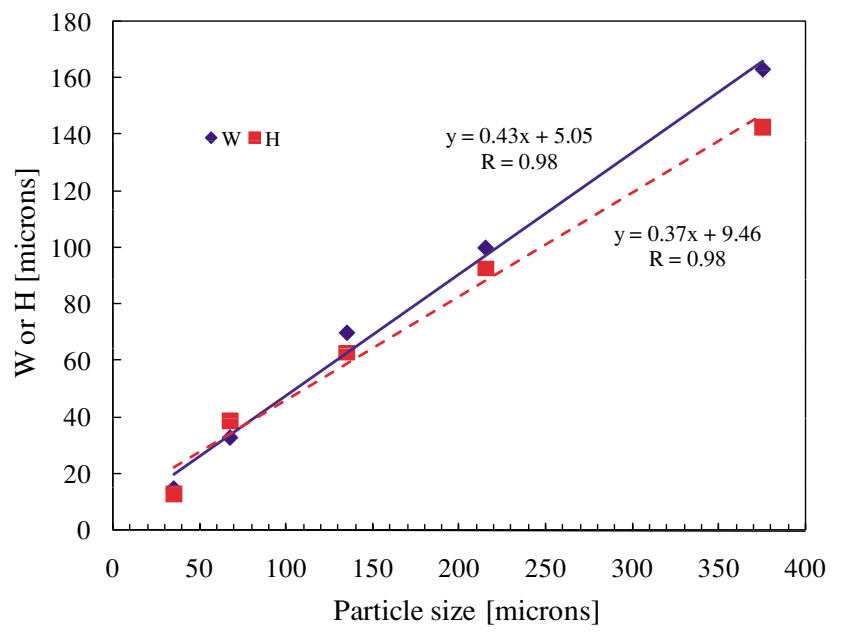

Fig. 2. The rectangular surface model seems to be the most suitable for experimental results with the half-wavelength and height, $W$ and $H$, respectively. Relationships of $W$ and $H$ against the particle size are found to be approximately linear, as shown in the graph.

We chose three rocks of olivine basalt, dacite, and dunite as representative specimens of fine to coarse grain size and mafic to silicic composition. We prepared the specimens by crushing half of these rocks and sieving them into five size categories of 25-45, 45-90, 90-180, 180-250, and 250$500 \mu \mathrm{m}$, respectively. A microscopic technique was used to measure the lengths of the long axis and its perpendicular axis for each particle of those specimens, using the geometric mean as the size of each particle. We obtained the cumulative mass distribution, which indicates the mass fraction of specimens smaller than a given particle size. For each category, the average size below which the cumulative mass fraction equals $50 \%$ of the total mass corresponds to $35,68,135,215$, and $375 \mu \mathrm{m}$, respectively. We also prepared flat plate specimens from the rest of these rocks.

Figure 2 shows an example of X-ray spectra obtained for dacite composed of 68 - and $215-\mu \mathrm{m}$ powders in comparison with those of the flat plate. Incident and emission angles are $55^{\circ}$ and $0^{\circ}$, respectively. The XRF lines of $\mathrm{Al}, \mathrm{Si}, \mathrm{Ca}, \mathrm{Ti}$, and $\mathrm{Fe}$ and the scattered lines of $\mathrm{Cr}$ are clearly detectable. A decrease in XRF intensities was apparent for the rougher surface specimens, which is more remarkable for Si than for $\mathrm{Fe}$, implying the trend is more effective for lower energy. 


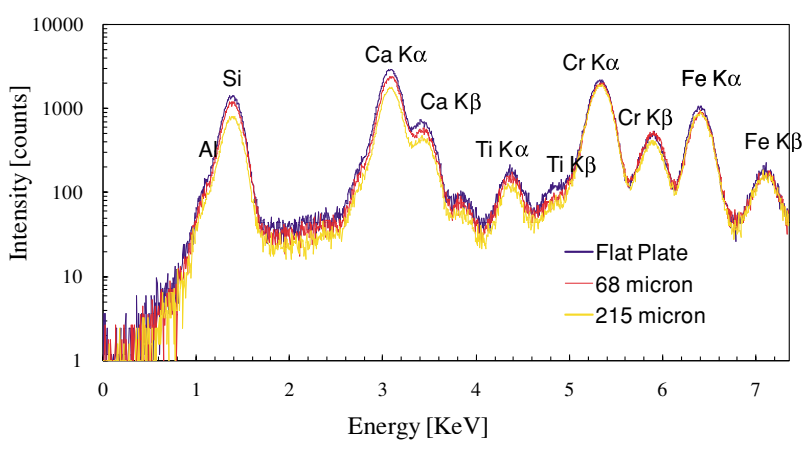

Fig. 3. X-ray spectra of dacite specimens are compared with different surface conditions of flat, $68 \mu \mathrm{m}$, and $215 \mu \mathrm{m}$, respectively. XRF lines of $\mathrm{Al}, \mathrm{Si}, \mathrm{Ca}, \mathrm{Ti}$, and $\mathrm{Fe}$ are clearly detectable, as are scattered lines of Cr. The XRF intensities can be seen to decrease more effectively for a rougher (larger particle size) surface, and the trend is more remarkable for lighter elements. For example, the decreases of $\mathrm{Si}-\mathrm{K} \alpha(1.74 \mathrm{KeV})$ and $\mathrm{Fe}-\mathrm{K} \alpha(6.40 \mathrm{KeV})$ correspond to 0.86 and 0.89 in the case of $68 \mu \mathrm{m}$, and to 0.67 and 0.85 in the case of $215 \mu \mathrm{m}$, respectively.

In this study, we focus on the experimental results of $\mathrm{XRF}$ intensities conducted under a variety of incidence angles and a constant emission angle $\left(e=0^{\circ}\right)$, with the aim at simulating the observation by the XRS onboard the SELENE orbiter.

\section{Surface Modeling and Numerical Calculations}

We also performed numerical calculations and compared them with experimental results to investigate the particle size effect in XRF. We attempted to explain this effect as being due to the shadowing of incident X-rays and the shielding of fluorescent X-rays by surface roughness. As was introduced in previous studies (Kuwada et al., 1997), we made a surface roughness model using a twodimensional rectangular wave form characterized by halfwavelength and amplitude, $W$ and $H$, respectively. The wave form is simple, with only two parameters used, but it has been found to be approximate experimental results more closely than any other forms, such as triangular or sine curves, which have critical incident or emission angles corresponding to the inclination of the steepest slope.

Two-dimensional surface profiles of the powdery specimens were measured using a scanning laser microscope (KEYENCE Violet Laser VK-9700). The small-scale roughness and offset slope are smoothed from these data with an appropriate bandpass filter to derive the averaged height-distance curves. The relationship of parameters, $W$ and $H$, on the typical particle size $D$ was found to be almost linear (Fig. 3). The linear fits of these as a function of particle size are $W=0.429 D+5.051$, and $H=$ $0.367 D+9.464$, respectively, for $25 \mu \mathrm{m}<D<500 \mu \mathrm{m}$. We then conducted the numerical studies using the relation.

We present here the method of numerical calculations for the rectangular surface model corresponding to the specimen's particle size. Let us suppose that given the configuration shown in Fig. 4, at incident and emission angles, $i$ and $e$, respectively, the intensities of fluorescent and scattered $\mathrm{X}$-rays can be calculated when the intensities and spectral profiles of the incident X-rays are assumed and the composition of the specimens are given. At the local point $\mathrm{P}$ inside

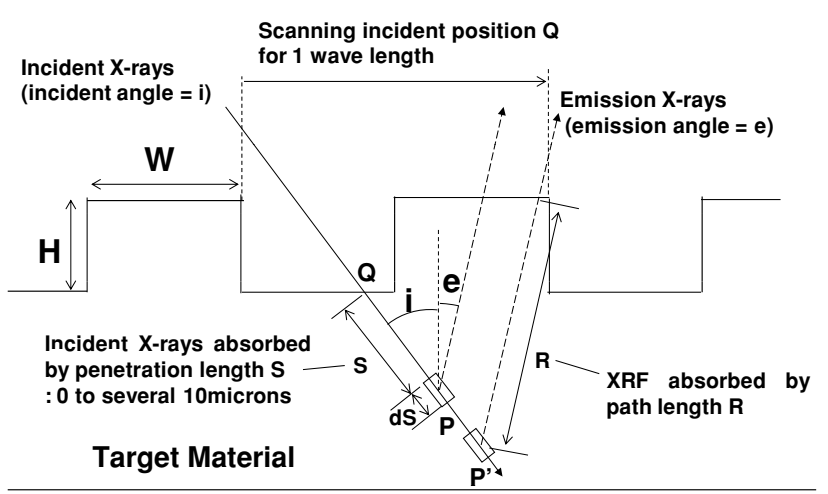

Fig. 4. Schematic diagram of the numerical studies for X-ray fluorescence with rectangular form with half-wave length $W$ and height $H$ are shown for incident and emission angle of X-rays, $i$ and $e$, respectively.

the material, incident X-rays to the point are absorbed by the material along the length of the penetration path. The fluorescent and scattered X-ray intensities excited by the absorbed incident $\mathrm{X}$-rays are calculated using the common $\mathrm{X}$-ray tables. The emission intensities at the surface are derived by considering the absorption along the path length betweene point $\mathrm{P}$ and the surface. The intensities excited by the incident $\mathrm{X}$-rays at a surface point $\mathrm{Q}$ are then calculated by the integration of those intensities emitted at various points along the penetration path. Therefore, the total intensities are calculated by scanning the incident point for a wave length of the rectangular wave form.

\section{Results of Particle Size Effect on XRF Intensi- ties}

We present here the experimental and numerical results focusing on the planned XRS observation from the SELENE orbiter. We first show the dependency of particle size on XRF intensities. In Fig. 5, XRF intensities of Si excited from powdery specimens (olivine basalt) normalized to those from the original flat rock plates are shown as a function of particle size. The diamonds indicate the experimental results at incident angles ranging from $35^{\circ}$ to $75^{\circ}$ with a constant emission angle of $0^{\circ}$, which corresponds to the direction perpendicular to the surface of specimen. The lower and upper limits of the error in size indicate those particle sizes below which the cumulative mass fractions equals 20 and $80 \%$ of the total mass, respectively. The error in size is shown only in the case of $i=35^{\circ}$, but it is the same at other incident angles. The error in intensity ratio shows the 3-sigma range of the statistical error of the fittings for the XRF line spectra. Lines denote the numerical calculation results under the same conditions. It should be noted that both the experimental and numerical results are in good agreement for any particle size and incident angle. The relative XRF intensities decrease with increasing average particle size, then slowly level off. These phenomena are found more effectively at larger incident angles, indicating that the apparent decrease in XRF intensities will be found near the terminator or at high latitudes.

The dependency of each major element on XRF intensities is shown in Fig. 6 for the powdery specimens (olivine 


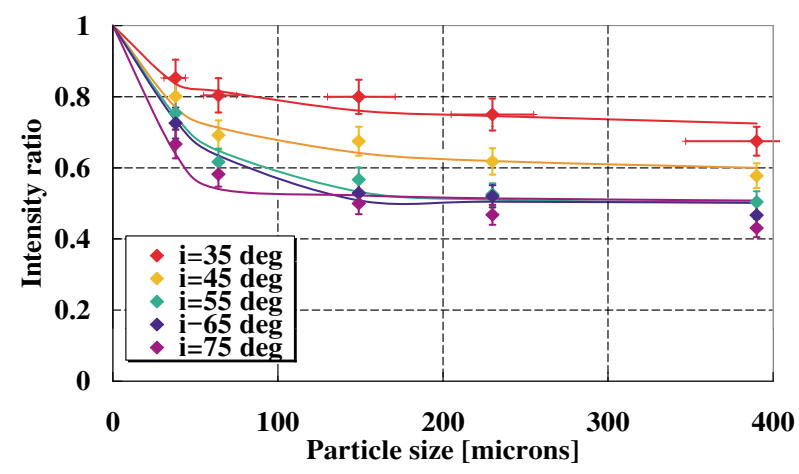

Fig. 5. XRF intensities of Si for crushed and sieved powdery sample (olivine basalt) normalized to those observed for original flat rock sample are shown as a function of particle size. Solid diamonds denote experimental results while the lines show numerical results. Emission angles were fixed to $0^{\circ}$, with the incident angles varying from $35^{\circ}$ to $65^{\circ}$.

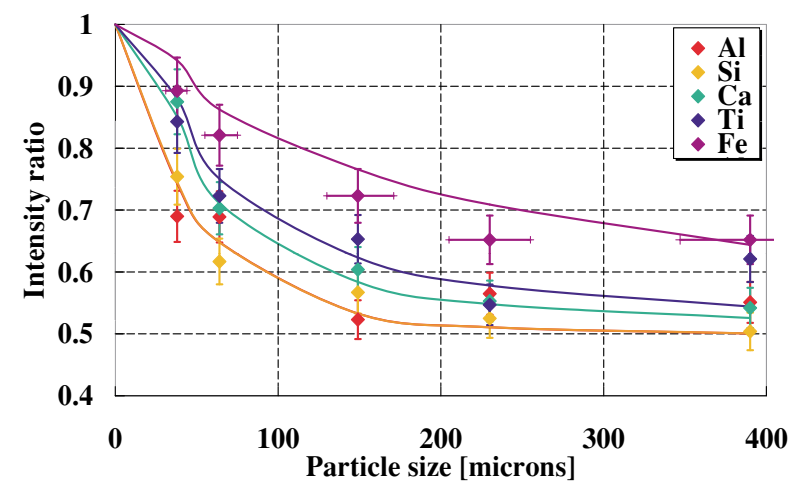

Fig. 6. The same as in Fig. 5, but the plots are shown for five major elements when observed with fixed emission and incident angles at $0^{\circ}$ and $65^{\circ}$, respectively. The lighter elements are more effective in decreasing XRF intensity, but the ratio apparently levels off at about 0.5 .

basalt) with incident and emission angles of $55^{\circ}$ and $0^{\circ}$, respectively. The diamonds and lines denote the experimental and numerical results, respectively, as in Fig. 5. Some of the data from the experimental and numerical results do not agree, such as Al data, due to low signal-to-background ratios. Although a similar trend of a decrease in the XRF intensity ratio with increasing particle size can be seen in each element, the lighter elements have a steeper decline at a smaller particle size and also show smaller intensity ratios at any particle size investigated here. Thus, these phenomena are apparently more effective at lighter elements. Again, the experimental and numerical results show in good agreement with each other. We confirmed that these results basically hold good for other incident angles and other rock specimens.

The dependency of each element on XRF intensities is the most important problem. For example, in Fig. 6, the intensity ratios of $\mathrm{Fe}$ and $\mathrm{Si}$ at $68 \mu \mathrm{m}$ are 0.82 and 0.61 , respectively. The intensity ratio of $\mathrm{Fe} / \mathrm{Si}$ is then more than $30 \%$ higher than expected for flat plate, and this could cause a severe error for any quantitative major elemental analysis without any corrections.

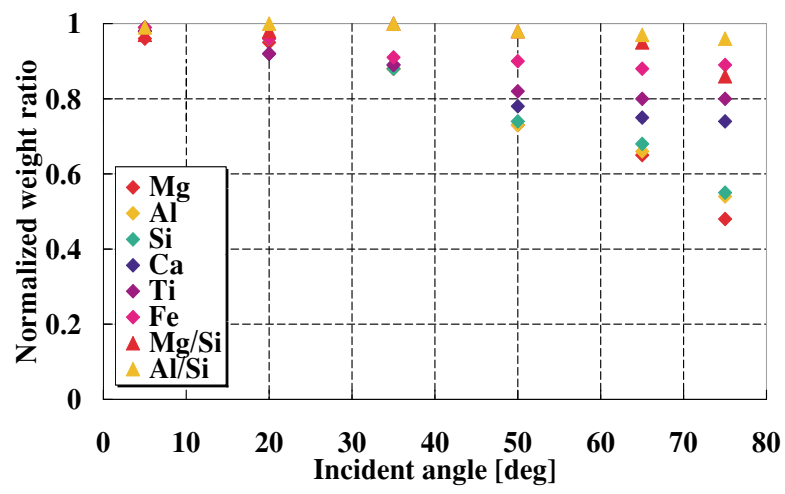

Fig. 7. Numerical estimation of relative XRF intensities from planetary surface (average grain size is $75 \mu \mathrm{m}$ and typical soil composition at Apollo 12 site is assumed) normalized by those from a flat surface is shown. The excitation source is the solar X-rays calculated using the equations in Mewe et al. (1986), dominantly generated by thermal bremsstrahlung emission at $8 \mathrm{MK}$ with line emissions by the collision process. Emission angles were fixed to $0^{\circ}$, with the incident angle varying from $5^{\circ}$ to $75^{\circ}$.

\section{Implications to Lunar XRF Spectrometry}

The lunar surface is covered with regolith whose typical particle size is $50-100 \mu \mathrm{m}$ when we take the size below which $50 \%$ of mass are included in cumulative size distribution of lunar regolith (Heiken et al., 1991). Like the Moon, the surface of planets and relatively large asteroids is generally expected to contain regolith on the uppermost surface due to impact ejecta sedimentation. Thus, the effects of particle size and incident angle should require corrections for quantitative elemental analysis by remote XRF spectrometry.

We believe that the numerical calculation with our rectangular surface model has been proven to be able to explain the particle size effect in the XRF intensity ratio within a $10 \%$ error, as shown in Figs. 5 and 6. Therefore, we apply our numerical studies to remote lunar XRF spectrometry, especially for the XRS onboard the SELENE orbiter. The SELENE is a three-axis stabilized satellite orbiting the Moon in a polar circular orbit (Sasaki et al., 2003). The attitude will be controlled for remote instruments to point at the lunar surface in the nadir direction. Thus, the XRS observation will be conducted under various incident angles of solar X-rays and constant XRF emission angle of $0^{\circ}$.

Suppose a typical solar X-ray intensity and spectral profile of a C-class flare is considered, and the temperature of solar coronal regions is assumed to be $8 \mathrm{MK}$ (Mewe et al., 1986; Ogawa et al., 2008). Assuming a surface elemental composition of soils similar to the Apollo 12 site (Heiken et al., 1991), we estimated relative XRF intensities with phase angle varying from $35^{\circ}$ to $75^{\circ}$. Figure 7 shows the calculated XRF intensities from the powdery planetary surface normalized to that of flat surface. As seen in the laboratory experiment, calculations also show a clear decrease in the absolute XRF intensities for larger phase angles. Such a trend is more remarkable for lighter elements. The XRFnormalized intensities of almost all of the major elements except for Fe decrease by more than $10 \%$ with increasing incident angle. For example, XRF-normalized intensities of Si are 0.89 at $i=35^{\circ}, 0.74$ at $i=50^{\circ}$, and 0.56 at $i=75^{\circ}$, 
respectively. This means higher abundance ratios for $\mathrm{Fe} / \mathrm{Si}$ or $\mathrm{Ca} / \mathrm{Si}$ would be estimated if no incident angle-dependent corrections are applied. This is a substantial problem for rock-type classification by means of remote lunar and planetary XRF spectrometry.

\section{Concluding Remarks}

Our experimental results on the particle size effect in XRF show a clear decrease in XRF intensities when these are observed at larger particle size and larger incident angle. The trend is more prevalent in lighter elements so that any elemental analysis of $\mathrm{Fe} / \mathrm{Si}$ or $\mathrm{Ca} / \mathrm{Si}$ might be misled by higher values without any corrections for incident angle or considerations for particle size effect in XRF. We also conducted numerical studies to simulate the effects by assuming the surface roughness as rectangular wave functions. Our experimental and numerical results are in good agreement with each other. We thus extended our numerical studies to apply for remote planetary XRF spectrometry, especially in the case of the XRS observation on the SELENE orbiter, where the emission angle is always $0^{\circ}$. Similar conclusions can be drawn from these results as those from the laboratory experiments - that the particle size effect in XRF cannot be neglected and should be corrected for quantitative major elemental analysis, especially when observed at large phase angles. Similar conditions will occur in future remote planetary XRF experiments, such as the Chang'E-1 and Chandrayaan-1 lunar orbiter missions (Goswami et al., 2006), and Messenger and Bepi Colombo mercury orbiters (Starr et al., 2001; Benkhoff, 2007).

Further studies are required to investigate this phenomenon under various conditions, both experimentally and numerically, and to formulate the effects mathematically. More realistic surface modeling should be also considered in the future.

Acknowledgments. The authors would like to express their thanks to anonymous reviewers for helpful comments, detailed discussions, and corrections of English to improve this manuscript. The authors are grateful to the SELENE XRS team for constructive discussion and cooperation. This work is partly supported by Grant-in-Aid for Young Scientists-A.

\section{References}

Abdunnabi, A. R. and M. Wasilewska-Radwanska, Study of the influence of geometry and energy of primary X-rays on the grain size effect in $\mathrm{XRF}$ analysis of intermediate thickness samples, Appl. Radiat. Isot., 45, 233-238, 1994.

Adler, I. J. and J. I. Trombka, Orbital chemistry: lunar surface analysis from X-ray and gamma-ray remote sensing experiments, Phys. Chem. Earth, 10, 17-43, 1977.

Benkhoff, J., MPO - the Bepi Colombo Mercury Planetary Orbiter, Lunar Planet. Sci., 38, 2169, 2007.

Claisse, F. and C. Samson, Adv. X-ray Anal., 5, 335-354, 1962.

Goswami, J. N., K. Thyagarajan, and M. Annadurai, Chandrayaan-1: Indian mission to the ,Lunar Planet. Sci., 37, 1704, 2006.

Grande, M., B. J. Kellett, C. Howe, C. H. Perry, B. Swinyard, S. Dunkin, J. Huovelin, L. Alha, L. C. D’Uston, S. Maurice, O. Gasnault, S. Barabash, K. H. Joy, I. A. Crawford, D. Lawrence, V. Fernandes, I. Casanova, M. Wieczorek, N. Thomas, U. Mall, B. Foing, D. Hughes, H. Alleyne, S. Russell, M. Grady, R. Lundin, D. Baker, C. D. Murray, J. Guest, and A. Christou, Observations of past lunar landing sites by the D-CIXS X-ray spectrometer on SMART-1, Lunar Planet. Sci., 38, 1154, 2007.

Heiken, G. H., D. T. Vaniman, and B. M. French, Lunar sourcebook: $a$ user's guide to the Moon, pp. 736, Cambridge University Press, 1991.
Kellett, B. J., M. Grande, and the D-CIXS Science Team, X-ray fluorescence observations of the Moon: Highlights from the first year of observations from D-CIXS on SMART-1, Lunar Planet. Sci., 37, 1897, 2006.

Kuwada, Y., Particle size effects in X-ray fluorescence spectrometry, MSc. Thesis, Aoyama Gakuin University, pp. 67, 1998.

Kuwada, Y., T. Okada, and H. Mizutani, Particle size effects in X-ray fluorescence spectrometry, in Proc. ISAS Lunar Planet. Symp., edited by Mizutani, H. and M. Kato, ISAS, Sagamihara, 30, 212-215, 1997.

Maruyama, Y., X-ray fluorescence experiments at rough surface simulating lunar and planetary surfaces, MSc Thesis, University of Tokyo, pp. 150, 2007.

Maruyama, Y., K. Shirai, Y. Yamamoto, T. Okada, and M. Kato, Laboratory experiments of X-ray fluorescence simulating lunar and planetary rough surface, in Proc. ISAS Lunar Planet. Symp., edited by Kato, M. and S. Tanaka, ISAS, Sagamihara, 38, 2006 (in press).

Maruyama, Y., K. Ogawa, T. Okada, and M. Kato, Particle size effect in $\mathrm{X}$-ray fluorescence and its implication to planetary XRF spectroscopy, Lunar Planet. Sci., 38, 1186, 2007.

Mewe, R., J. R. Lemen, and G. H. J. van den Oord, Calculated X-radiation from optically thin plasmas: VI. Improved calculations for continuum emission and approximation formulae for nonrelativistic average Gaunt factors, Astoron. Astorophys. Suppl., 65, 511-536, 1986.

Näränen, J., H. Parviainen, and K. Muinonen, X-ray fluorescence modeling for solar system regoliths: effects of viewing geometry, particle size, and surface roughness, in Proc. International Astronomical Union, Vol.2, Symp. S236, Near-Earth Object, our celestial neighbors: Opportunity and risk, edited by Milani, A., G. Valsecchi, and D. Vokrouhlicky, Cambridge Univ. Press, 243-250, doi: 10.1017/S1743921307003298, 2007.

Nielson, K. K. and V. C. Rogers, Particle size effects in geological analyses by X-ray fluorescence, Adv. X-ray Anal., 29, 587-592, 1986.

Nittler, L. R., R. D. Starr, L. Lim, T. J. McCoy, T. H. Burbine, R. C. Reedy, J. I. Trombka, P. Gorenstein, S. W. Squyres, W. V. Boynton, T. P. McClanahan, J. S. Bhangoo, P. E. Clark, M. E. Murphy, and R. Killen, X-ray fluorescnece measurments of the surface elemental composition of asteroid 433 Eros, Meteorit. Planet. Sci., 36, 1673-1695, 2001.

Ogawa, K., T. Okada, K. Shirai, and M. Kato, Numerical estimation of lunar X-ray emission for X-ray spectrometer onboard SELENE, Earth Planets Space, 60, this issue, 283-292, 2008.

Okada, T., Particle size effect in X-ray fluorescence at a large phase angle: importance on elemental analysis of asteroid Eros (433), Lunar Planet. Sci., 35, 1927, 2004.

Okada, T., M. Kato, Y. Yamashita, K. Shirai, Y. Yamamoto, T. Matsuda, H. Tsunemi, and S. Kitamoto, Lunar X-ray spectrometer experiment on the SELENE mission, Adv. Space Res., 30, 1909-1914, 2002.

Okada, T., K. Shirai, Y. Yamamoto, T. Arai, K. Ogawa, K. Hosono, and M. Kato, X-ray fluorescence spectrometry of Asteroid Itokawa by Hayabusa, Science, 312, 1338-1341, 2006.

Okada, T., K. Shirai, Y. Yamamoto, T. Arai, K. Ogawa, T. Inoue, and M. Kato, Elemental composition of asteroid Itokawa by Hayabusa XRF spectrometry, Lunar Planet. Sci., 38, 1287, 2007.

Sasaki, S., Y. Iijima, K. Tanaka, M. Kato, M. Hashimoto, H. Mizutani, and Y. Takizawa, The SELENE mission: Goals and status, Adv. Space Res., 31, 2335-2340, 2003.

Shirai, K., T. Okada, Y. Yamamoto, T. Arai, K. Ogawa, H. Shiraishi, M. Iwasaki, M. Arakawa, M. Grande, and M. Kato, Instrumentation and performance evaluation of the XRS on SELENE orbiter, Earth Planets Space, 60, this issue, 277-281, 2008.

Starr, R. D., G. C. Ho, C. Schlemm, R. E. Gold, J. O. Goldsten, W. V. Boynton, and J. I. Trombka, The X-ray spectrometer for Mercury Messenger, in Proc. Workshop on Mercury: Space environment, surface, and interior, edited by Robinson, M. and G. J. Taylor, LPI., 102, 2001.

Suortti, P., Effects of porosity and surface roughness on the X-ray intensity reflected froma powder specimen, J. Appl. Cryst., 5, 325-331, 1972.

Trombka, J. I., S. W. Squyres, J. Brueckner, W. V. Boynton, R. C. Reedy, T. J. McCoy, P. Gorenstein, L. G. Evans, J. R. Arnold, R. D. Starr, L. R. Nittler, M. E. Murphy, I. Mikheeva, R. L. McNutt, Jr., T. P. McClanahan, E. McCartney, J. O. Goldsten, R. E. Gold, S. R. Floyd, P. E. Clark, T. H. Burbine, J. S. Bhangoo, S. H. Bailey, and M. Petaev, The elemental composition of asteroid 433 Eros: Results of the NEAR-Shoemaker Xray Spectrometer, Science, 289, 2101-2105, 2000.

Y. Maruyama, K. Ogawa, T. Okada (e-mail: okada@planeta.sci.isas. jaxa.jp), and M. Kato 